\title{
Botulinum Toxin Therapy for Involuntary Facial Movement
}

\section{J. S. ELSTON}

\section{London}

The two principal involuntary facial movement disorders under discussion in this paper are blepharospasm and hemifacial spasm. Blepharospasm is a focal dystonia involving the orbicularis oculi, presenting insidiously in the sixth and seventh decades, with a female preponderance. It usually progresses to cause severe visual disability, and the dystonic spasms may also involve the lower face, the jaw and neck muscles (oro-mandibular dystonia), and other cranially innervated muscles (segmental dystonia). ${ }^{1}$ It is due to organic dysfunction of the basal ganglia and may occur in generalised movement disorders such as Parkinson's disease and progressive supranuclear palsy. The condition responds poorly to systemic drug treatment, but well to denervation of the orbicularis by facial nerve avulsion. The operation is, however, cosmetically disfiguring and the facial nerve frequently regenerates to cause recurrent spasm. ${ }^{2}$ Local, albeit temporary, denervation with botulinum toxin is therefore an attractive alternative.

Hemifacial spasm typically begins around the eye and subsequently spreads into the ipsilateral 7th nerve innervated muscles, including the platysma. Electrophysiological investigation and neurosurgical operative findings indicate that it is due to irritation of the facial nerve by an aberrant blood vessel in the posterior fossa, and the movement control systems are otherwise normal. ${ }^{3}$ Drug treatment is seldom helpful; neurosurgery can be curative, but may rarely cause a permanent facial palsy or deafness, and the condition may be recurrent. The treatment potential of botulinum toxin is clear.

A number of other involuntary facial movement disorders, such as aberrant regeneration of the facial nerve after a palsy, may also be expected to respond well to local denervation.

\section{Patients, Materials and Methods}

A total of 243 patients have been treated: 194 with blepharospasm (see Table I), 42 with hemifacial spasm and 7 with other abnormal movements. All cases have been followed up for at least 5 months or 2 sets of injections.

$0.05 \mu \mathrm{g}$ freeze-dried botulinum toxinhaemagglutinen complex $\quad(0.008 \mu \mathrm{g}$ neurotoxin) was reconstituted with $1 \mathrm{ml}$ of sterile saline and then diluted in a further $9 \mathrm{ml}$ saline. For the first treatinent for blepharospasm, 2 injections of $0.2 \mathrm{ml}$ of this solution were given subcutaneously laterally and $0.1 \mathrm{ml}$ medially, at the junction of the orbital and preseptal orbicularis oculi in the upper and lower lids of both eyes. For hemifacial spasm and other unilateral disorders the same dose was used on the affected side. Subsequent treatments were modified according to the response-usually half the starting dose was needed to maintain the muscle weakness.

The patients' visual function before treatment was assessed and graded from 1-6 (see Table II), the same scale being used to assess the effects of treatment.

In the treatment of hemifacial spasm (42 cases) and other unilateral facial movement disorders ( 7 cases), the patients assessed the percentage reduction in abnormal movements following injections (Table III).

\section{Results \\ Blepharospasm}

Ninety per cent of the patients were improved by the treatment, and 72 per cent became 
Table I Clinical findings in 194 patients with visually disabling blepharospasm

\begin{tabular}{lll}
\hline Blepharospasm alone & 38 & $(19.5)^{*}$ \\
plus lower facial movements & 31 & $(16)$ \\
plus oromandibular dystonia & 59 & $(30.5)$ \\
plus segmental dystonia & 48 & $(25)$ \\
plus generalised movement & & \\
$\quad$ disorder & 18 & $(9)$ \\
\hline
\end{tabular}

*Figures in brackets indicate percentage of total.

Table II Blepharospasm: grading of visual status

\begin{tabular}{ll}
\hline & Category \\
\hline Functional blindness & 1 \\
Dependent outside home & 2 \\
Independent: poor function & 3 \\
Independent: moderate function & 4 \\
Inconvenienced & 5 \\
Normal & 6 \\
\hline
\end{tabular}

Table III Hemifacial spasm and other unilateral facial movement disorders: $n=49$

\begin{tabular}{ll}
\hline Hemifacial spasm: 42 cases & \\
Female & 22 \\
Male & 20 \\
Right side & 17 \\
Left side & 25 \\
Onset age & 51.9 \\
Previous treatment: & \\
Drugs & 31 \\
Posterior fossa surgery & 5 \\
Peripheral surgery & 2 \\
\hline Other disorders: 7 cases & \\
Abberrant regeneration after facial & \\
$\quad$ palsy & 5 \\
Unilateral lower facial dystonia & 2 \\
\hline
\end{tabular}

either normal or only mildly inconvenienced by their condition (Fig. 1). In most of the 10 per cent who failed to respond, orbicularis weakness was adequate, but abnormal movements-Bell's phenomenom and levator palpebrae inhibition-persisted. The patients' age, and sex, the severity and duration of the blepharospasm, and the involvement of other muscles had no influence on the response, or the quality and duration of the improvement, which averaged 10 weeks. After facial nerve avulsion however, normal or near normal visual function was usually restored for an average of 17 weeks. An unexpected benefit was reduction in lower facial movements in 19 out of 31 cases and of oro-mandibular dystonia in 42 out of 59 cases.

Temporary, partial unilateral or bilateral ptosis occurred in 44 per cent of the patients treated at some stage, but more serious side effects were unusual, and only 5 patients declined more than 2 injections on this ground (Table IV). Ninety per cent continued with injection treatment, sometimes in combination with drugs or limited surgical treatment such as levator muscle advancement.

\section{Hemifacial spasm}

The average reduction in abnormal movements after the injections was 75 per cent, and lasted 15 weeks. A temporary partial ptosis occurred in 9 cases ( 21 per cent) and lower facial weakness, affecting upper lip mobility in 5 (12 per cent). Forty-one patients have continued with the treatment, one has opted for posterior fossa surgery.

All cases of aberrant regeneration and unilateral focal lower facial dystonia responded well, for an average of 18 weeks.

\section{Discussion}

The relief of the major symptoms of hemifacial spasm by selective weakening of involved muscles is readily understandable; the same applies to aberrant regeneration. In blepharospasm however, the abnormal movements are centrally determined, and more complex; forceful eye closure is associated with Bell's phenomenom of upward (or rarely downward) eye movement, and inhibition of the levator palpebrae. Surgical or chemical

Table IV Major side effects following treatment for blepharospasm (average duration 2.6 weeks): 194 cases

\begin{tabular}{lrr}
\hline Ptosis: Partial unilateral & 38 & $(10)^{*}$ \\
\multicolumn{1}{c}{$\quad \begin{array}{c}\text { Complete unilateral } \\
\text { bilateral }\end{array}$} & 47 & $(15)$ \\
bilateral & 1 & \\
Diplopia & 12 & $(3)$ \\
Entropion & 8 & \\
Lower facial weakness & 5 & \\
Blurred vision & 3 \\
\hline
\end{tabular}

*Figures in brackets denote cases where side effects have been recurrent. 


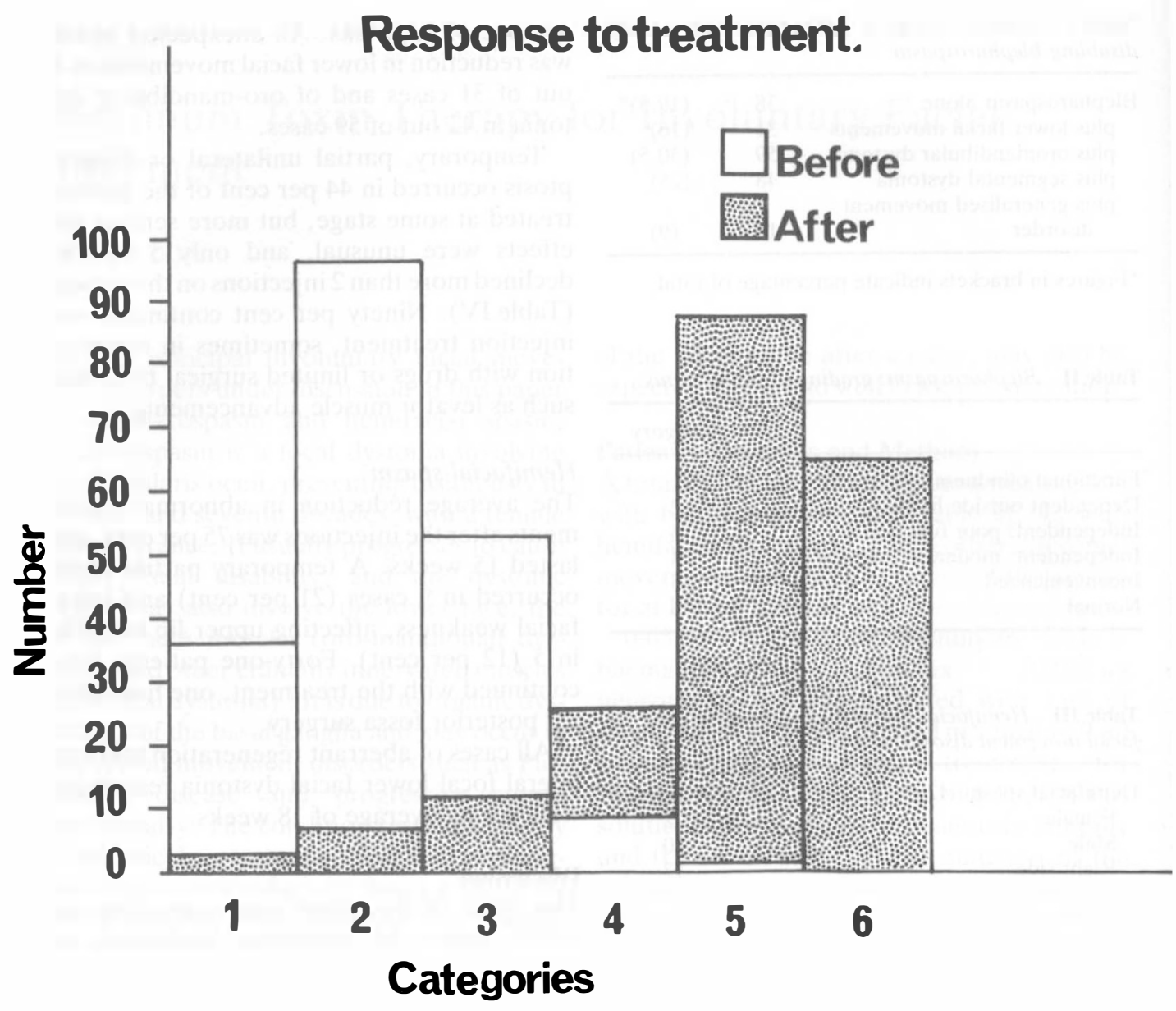

Fig. 1. Response to treatment with botulinum toxin injections 194 patients

denervation of the orbicularis abolishes the blepharospasm, but if it has no effect on the other components, the patient remains functionally blind, as in 10 per cent of those treated. In a further 58 per cent, vision was improved, but still to some extent compromised by abnormal movements, and only 32 per cent regained normal function. A previously successful facial nerve avulsion with re-innervation was the only predictor of a good response.

The mechanism for the complete resolution of the abnormal movements in one third of cases is unclear. One possibility is that the toxin may effect the intrafusal muscle fibres in the proprioceptive spindles of the treated orbicularis, modifying spindle afferent discharges and thereby facial nucleus motor neurone function. Peripheral treatment could thereby modify central nervous system function and, brain stem interneurone discharges could also be affected, allowing the Bell's phenomenom and levator inhibition to resolve.

Recent investigations into the physiology of botulinum toxin suggest another explanation. Retrograde intra-axonal transport of the toxin to the motor nerve cell body has been demonstrated, and the molecule could therefore directly affect facial nuclear function, and via interneurones, the whole movement complex. This mechanism could also help to explain the reduction in lower facial, jaw and 
neck movements in some patients. The variability in response may reflect the severity of the underlying abnormality of movement control, which may not equate with the physical signs.

Clearly, collaborative investigation between clinicians and physiologists must continue to establish the exact way in which botulinum toxin acts to modify the various abnormal facial movement disorders. The results presented, however, clearly demonstrate that botulinum injections should now be regarded as the treatment of choice in idio- pathic blepharospasm, hemifacial spasm and misdirection regeneration of the facial nerve.

\section{References}

1 Jankovic J and Ford J: Blepharospasm and orofacialcervical dystonia: Clinical and pharmacological findings in 100 patients. Ann Neurology 1983, 13: 402-11.

${ }^{2}$ Bird AC and McDonald WI: Essential Blepharospasm. Trans Ophthalmol Soc UK 1975, 95: 250-3.

${ }^{3}$ Auger RG, Pipgras PG, Laws ER, Miller RH: Microvascular decompression of the facial nerve for hemifacial spasm: clinical and electrophysiological observations. Neurology 1987, 31: $346-50$. 